\title{
Complicaciones de ventilación mecánica en niños
}

\author{
Silvia Arriagada T. ${ }^{1}$; Jaime Cordero T, ${ }^{1}$; José Baeza R.+ ${ }^{1}$ \\ Complications of mechanical ventilation in children
}

\begin{abstract}
Morbidity and lethaliy associated to mecharica venttation were prospectively recorded in 294 children who equired 300 consecutive artific al respiration procedures. Fourteen complications were previously defined, including those related to intstation, exlubction. endorrocheal or rocheostomy tube. venti ator failure, jogether with medical complications. The meon age of these patients was 20 monitis irange 1 month 1014 years). Average duration of ventilatory support was 0.32 days, range one hour to 35 doys. One or more complications were recorded in 160 cases $[55.3 \%$, the rast frequen being righ mainstem bronchus intubat on $11.6 \%$. self extubation $111.6 \%$. endotracheal tube obstruction $\{11.3 \%$, rosocomial oneumonia $\$ 9.3 \%$, prolonged intubation attempt $10.0 \%\}$ and barctrauma $10.6 \%$. Whale lel zality rate was $32.6 \%, 96$ patientși. being difectly related to mechanical ventilation in seven coses: in two polients it was due to ube obsirciclion, in one child to oarotrcumo, in anothes one to massive otelectasis and in three intants less tha" one year old with complex card ac songenital defects and long periods of mechanical vent lation, to nosccomial pneumonia.
\end{abstract}

(Key words: respiration, artific cl, ventilatio-, mechanical.)

En las unidades de tratamiento intensivo la ventilación mecánica es un recurso fundamental para los pacientes con falla respiratoria de origen pulmonar o extrapulmonar. La proporción de los enfermos que requieren ventilación mecánica en UTI yaría de 18 a $80 \%$, según las características de cada centro. El procedimiento implica complicaciones y efectos adversos, que pueden interferir directa 0 indirectamente en la sobrevida o prolongar su empleo. La letalidad relacionada con la ventilación mecánica varía con la enfermedad de base, entre 20 y $36 \%$, siendo atribuible directamente a complicaciones alrededor de $2,8 \%{ }^{1-8}$.

El objeto de este análisis fue describir la letalidad y la incidencia de complicaciones en pacientes sometidos a ventilación mecánica desde el momento de la entubación, durante la ventilación mecánica y el período de desconexión, con el fin de identificar hechos que permitan mejorar la atención de ellos.

\section{Pacientes y Métodos}

Eolre el 1 de enero de 1988 y el 31 de mayo de 1989 ingresaron a la unidad de tratarniento intensivo del Hospi-

1. Unidad de Cuidedos Intensivos, Hospital Luis Calvo Mackenna. tal Luis Calyo Mackenna 633 pacientes, de los cuales 294 $(46,4 \%)$ requirieron 300 episodios consecutivos de ventilación mecánica que se estudiaron en forma prospectiva. En todos los casos se usaron ventiladores ciclados por tiempo y limitados por presión (Sechrist IV-100 B). Bear Cub 2001, Heatthdyne 105, Newport E lo0i, IMV Bird, (Baby Bird). Para el registro de ta información se diseñó una ficha que incluía los antecedentes clinicos, de laboratorio y las complicaciones (dependientes de la entubación y extubación; del tubo endotraqueal o la traqueotomía; el ventilador o médicas). Se consideró que la entubación fue prolongada cuando se realizaron más de tres intentos o éstos duraron más de tres minutos. Se definió como extubación prematura la que fue seguida de reentubación antes de transcurrir 12 horas. Se denomino filtración de] tubo cualquier fuga de aire producida por deficiente relación del ubo con la tráquea. que impidiese alcanzar la presión inspiratoria deseada con el ventilador y como obstrucción del mismo la aparicion de hipoxemia repentina o dificultad para introducir la sonda de aspiración. La lesión destructiva de piel y cartílago nasal por roce con el tubo endotraqueal se consignó como necrosis nasal. La traqueítis necrotizante sólo fue detectada en pacientes fallecidos. sometidos a necropsia, pues se definió por las alteraciones macroscópicas e histológicas en la tráquea. Entre las complicaciones dependientes del ventilador mecáni. co se consideraron la falla de las alarmas, desconexión del circuito, nebulizacion inadecuada que dificultase la aspiración de secreciones o facilitase la obstrucción del tubo, respirador mal armado y fatta de la máquina. Como complicaciones médicas se registraron los neumotórax, neumodiastino, enfisemas subcutaneos, fístulas broncopleurales y las atelectasias, todas confirmadas con radiografia de tórax frente a la sospecha clínica. Se denominó bipoventilación 
alveolar a la acidosis respiratoria con $\mathrm{pH}$ arterial menor a 7,30 y $\mathrm{PaCO}, 55$ o más durante la ventilación mecánica, que se corrigieron con solo modificar las reglas de los controles del ventilador. La neumonía se califico nosocomial si el paciente tenía más de 72 horas de ventilación mecánica, la radiografia đe tórax mostraba nuevas imágenes de condensación pulmonar y el hemograma era compatible con infección en un paciente con manifestaciones clinicas sugerentes. Las afecciones que provocaron la necesidad de ventilación mecánica fueron ctasificadas en médicas y quirúrgicas.

\section{Resultados}

Los 294 pacientes registrados en el tiempo del estudio requirieron 300 episodios de ventiIación mecánica. Su edad varió de un mes a 14 años (promedio 20 meses), $77,5 \%$ eran lactantes (de ellos $83 \%$ eran menores de un año), $6,8 \%$ preescolares y $15,6 \%$ escolares. Ciento sesenta y seis pacientes $(56,4 \%)$ eran varones. El promedio de la duración de la ventilación mecánica fue 6,32 días, márgenes de una hora hasta 85 días. Las enfermedades de base por las que fue necesario usar ventilación mecánica fueron, en $238(79,4 \%)$ episodios, médicas y en 62 (20,6\%) quirúrgicas (tabla 1)

Uno o más de los catorce tipos de complicaciones seleccionados para análisis se registraron en $166(55,3 \%)$ de los 300 episodios consecutivos de ventilación mecánica, por un total de 284 complicaciones. Las complicaciones dependientes de la entubación y extubación fueron 112 $(37,3 \%)$ en total, siendo las más frecuentes la entubación monobronquial y la autoextubación. De las $82(27,3 \%)$ complicaciones relacionadas con el tubo endotraqueal y la traqueostomía, fue más frecuente la obstrucción del tubo. La falla de la máquina del ventilador fue la complicación más frecuente en el rubro respectivo y la neumonía nosocomial, con la hipoventilación alveolar, y el barotrauma entre las complicaciones médicas (tabla 2).

La letalidad del conjunto de los pacientes estudiados fue $32,6 \%(96 / 294), 31,5 \%$ en lactantes $(72 / 228), 40 \%$ en preescolates $(8 / 20)$ y $34,7 \%$ en escolares (16/46). La proporción de pacientes que fallecieron aumentó progresivamente a medida que se prolongó el tiempo de ventilación mecánica, pues fue de 17,6\% (13/ 74) si se usó por uno a tres días, ante $30,3 \%$ $(23 / 76, \mathrm{~ns}) ; 43,5 \%(27 / 62, \mathrm{p}<0,002)$ y $64,7 \%$ (11/17, p $<0,001)$ niños ventilados por uno a
Tabla 1

Enfermedades causantes del empleo de ventilación mecánica

\begin{tabular}{lrr}
\hline & $\mathrm{n}$ & $\%$ \\
\hline Causas médicas & 238 & $\mathbf{7 9 , 4}$ \\
Vía aérea inferior & 116 & 38,6 \\
Cardiovascular & 39 & 13,0 \\
Centro respiratorio & 38 & 12,6 \\
Vía aérea superior & 20 & 6.6 \\
Neuromuscular & 13 & 4.6 \\
Caja torácica & 12 & 4.0 \\
Causas quirúrgicas & 62 & 20,6 \\
Cirugía cardíaca & 38 & 12,6 \\
Cirugía general & 24 & 8,0 \\
\hline Toral & 300 & \\
\hline
\end{tabular}

Tabla 2

Incidencia de complicaciones observadas durante la ventilación mecánica

\begin{tabular}{lrr} 
& n & $\%^{*}$ \\
\hline Dependientes de la intubación y extubación & 112 & $\mathbf{3 7 , 3}$ \\
Intubación monobronquial & 35 & 11,6 \\
Autoextubación & 35 & 11,6 \\
Extubación prematura & 22 & 7,3 \\
Entubación prolongada & 20 & 6,6
\end{tabular}

Dependientes del lubo endotraqueal o traqueostomía

Obsiruction

Sangramiento

Filtración

Necrosis nasal

Traquej́tis necratizante

$34 \quad 11,3$

$20 \quad 6,6$

$19 \quad 6,3$

10,3

$9 \quad 22,5 * *$

Dependientes del ventillador

196,3

Falla respirador

Circuito

$7 \quad 2,3$

Humectación

Alammas

Mal armado

$5 \quad 1,6$

$5 \quad 1,6$

I $\quad 0,3$

I $\quad 0,3$

Complicaciones médlcas

Neumonía nosocomial

Hipoventilación alveolar

Barotrauma

$71 \quad 23,6$

Atelectasia

9,3

8,0

6.6

155,0

* Porcentajes referidos a 300 episodios de ventijación mećnica.

* Porcentaje referido a 40 pacientes fallecidos con anatomía patológica. 
tres, cuatro a siete o más de veintidos días respectivamente. Hace excepción a lo anterior la muy alta letalidad entre los pacientes ventilados menos de 24 horas $(22 / 65 ; 33,8 \%)$-incluidos 16 niños que ingresaron a la unidad en paro cardiorrespiratorio o en choque irreversible- la que, sin embargo, también diferfa significativamente con la de uno a tres días $(\mathrm{p}<0,05)$ y la de más de 22 días de ventilación $(p<0,05)$. La mayor letalidad por causas se registró en pacientes con alteraciones del centro respiratorio $(47,4 \%)$ y cirugía cardiovascular $(39,5 \%)$, seguida por la de los afectados por enfermedades de la vía aérea inferior $(34,5 \%)$, cardiovasculares $(33,3 \%)$, neuromusculares $(23,0 \%)$, de la caja torácica y cirugía general $(16,7 \%$ cada una), y de la vía aérea superior $(5,0 \%)$. Sólo se encontraron diferencias estadísticamente significativas al comparar la letalidad de las dos primeras causas $\neg$ que no diferían entre sí- con las afecciones de vía aérea superior $(\mathrm{p}<0,03)$.

En 40 de los 96 niños fallecidos se realizó estudio de anatomía patologica y en nueve de ellos $(22,5 \%)$ se encontro traqueítis necrótica, complicación que fue evaluada sólo en estos pacientes. En tres pacientes, todos con malformaciones cardíacas complejas y largo tiempo en ventilación mecánica, la causa principal de muerte fue neumonía nosocomial.

\section{Comentario}

La ventilación mecánica, por ser una medida agresiva o aplicarse en situaciones apremiantes, puede causar complicaciones o trastornos jatrogénicos, cuyo conocimiento -con el de sus causas- puede ayudar a evitarlas, mejorando sus resultados. El tipo y número de complicaciones dependen, en cada centro, de las características de los pacientes, la experiencia del equipo humano y los medios con que cuenta. Las enfermedades que con mayor frecuencia motivaron el uso de ventilación mecánica fueron, en nuestros pacientes, las afecciones respiratorias bajas. La letalidad registrada en un tercio de estos casos puede atribuirse a la extensión de las neumonías y a la severidad de la insuficiencia respiratoria con que la mayoría de ellos ingresó a la unidad y es comparable con la registrada en otras experiencias. Lo mișmo sucedió con los pacientes cuyo trastorno fundamental era una alteración del centro respiratorio (incluyendo los que sufrían traumatismo encefalocraneano grave, meningitis y encefalitis con edema cerebral), que mostraron la letalidad más alta de la serie $^{4,8}$. En el caso de los pacientes sometidos a cirugía cardíaca, la letalidad corresponde a la de malformaciones cardfacas complejas, de mal pronóstico, en que la cirugía fue muchas veces paliativa, para superar en parte problemas pulmonares secundarios a la anomalía cardiovascular.

La elevada letalidad entre los pacientes tratados con ventilación mecánica por menos de 24 horas se explica porque la mayoría de los que murieron ingresaron a la unidad en paro cardiorespiratorio o en estado de choque irreversible, correspondiendo los restantes, preferentemente, a pacientes en postoperatorio inmediato. El aumento de la letalidad en coincidencia con la prolongación de la duración de la ventilación mecánica es similar en otras publicaciones ${ }^{4,6,8}$.

La alta incidencia de extubación espontánea. en los rangos altos en relación a otras experiencias -donde es entre 3 y $15 \%-5.9 .10$. pudo haberse debido a fallas en la sedación o relajacion del paciente o la fijación del tubo endotraqueal.

Las maniobras prolongadas de entubación, por dificultades en la instalación del tubo, fueron casi seis veces menos frecuentes que lo registrado por otros". Io que atribuimos a la experiencia de los médicos que las efectuaron. La introducción del tubo en el bronquio principal derecho no causó muertes, a diferencia de lo ocurrido a otros autores ${ }^{6}$, porque, a pesar de suceder con frecuencia, se identifić y resolvió râpidamente gracias a la tutina de tomar radiografía de tórax inmedjatamente después de cada entubación.

La obstrucción del tubo ${ }^{3,4,11}$ se debio principalmente a humectación inadecuada $\mathrm{y}$, en un caso con traqueostomía, a sangramiento. Esta complicación tuvo incidencia directa en la mortalidad en dos casos, por hemorragia y secreciones. Creemos que es fundamental hacer el manejo de secreciones con aire inspirado debidamente humectado, para evitar tapones mucosos y la necesidad de procedimientos frecuentes de aspiración

La traqueítis necrótica fue un hallazgo de anatomía patológica. La lesión ha sido señalada cono complicación de la ventilación a alta frecuencia, especialmente en recién nacidos y des- 
crita en $59 \%$ de 206 pacientes con anatomia patológica, habiéndosela relacionado con isquemia de la mucosa y submucosa por asfixia neonatal severa o falla circulatoria ${ }^{12-15}$. La ventilación mecánica podría contribuir a la isquemia. Por tratarse de pacientes con anatomía patológica no sabemos cuál habría sido el futuro de ellos en cuanto a secuelas de tráquea. La necrosis nasal se observó en pacientes entubados por más de 15 días por vía nasotraqueal. El daño se debe al roce del tubo y, para evitarlo, es importante preocuparse de la posición y fijación de éstes.

La incidencia de las complicaciones dependientes del ventilador, expresadas por empeoramiento brusco de la función respiratoria que se supera rápidamente al corregir el defecto del equipo o de su manejo, está en íntima relación con la experiencia del personal a cargo. El barotrauma tuvo, en estos casos, una frecuencia intermedia entre otras experiencias ${ }^{2,4,5-7}$. Los neumotórax se registraron en pacientes con bronconeumonía extensa que requirieron presiones elevadas (inspiratoria sobre $30 \mathrm{~cm}$ de $\mathrm{H}_{2} \mathrm{O}$ y positiva al final de la expiración sobre $10 . \mathrm{cm}$ de agua) en el ventilador, pero no incidieron directamente en la letalidad debida a su detección y resolución precoz. La menor incidencia de neumonía nosocomial que en otras series ${ }^{3,5-7,11,13}$ puede deberse a dificultad de precisar el momento de la sobreinfección y subregistro, pero estuvo directamente involucrada en la muerte de tres pacientes. La hipoventilación alveolar se observó especialmente en pacientes en que. por su enfernedad de base, la distensibilidad pulmonar estaba disminuida y se usaron ventiladores ciclados por presión en vez de volumen. Esto se corrigió modificando los regímenes del ventilador en cada caso. Las atelectasias podrian atribuirse a no contar con kinesiterapia todas las horas del día y, además, al empleo de ventiladores ciclados por presión. En un caso la muerte se relacionó directamente con una atelectasia masiva.

La ventilación mecánica es un medio fundamental en el manejo de pacientes en estado grave, sin embargo su empleo se asocia a importante morbilidad y eventual letalidad, por lo que conviene hacer análisis sistemáticos y periódicos de las complicaciones para conocer su incidencia, detectar y corregir situaciones de riesgo para el paciente y evaluar la calidad de nuestra atención.

\section{Resumen}

Se estudiaron durante 17 meses, 300 episodios consecutivos de ventilación mecánica en 294 pacientes. Se definieron previamente 14 lipos de complicaciones, incluyendo las dependientes de la entubación y extubación, el tubo endotraqueal, la traqueostomía, el ventilador y las complicaciones médicas. La edad de los pacientes varió de un mes a 14 años. La media de la duración de la ventilación mecánica fue 6,32 días (márgenes una hora y 85 días). Se registraron una o más complicaciones en 166 de 300 procedimientos $(55,3 \%)$. Las más frecuentes fueron entubación monobronquial $11,6 \%$; autoextubación $11,6 \%$; obstrucción del tubo endotraqueal $11.4 \%$; neumonía nosocomial $9,3 \%$ : procedimiento prolongado de entubación $6.6 \%$ y barotrauma $6,6 \%$. La letalidad global fue de $32,6 \%$ y en siete casos la complicación tuvo incidencia directa en ella: en dos pacientes por obstrucción del tubo endotraqueal, en uno por barotrauma, uno por atelectasia masiva pulmonar $y$ tres por neumonía nosocomial. Los últimos tres niños eran menores de un año, con cardiopatías congénitas complejas y con tiempo de ventilación mecánica prolongada.

(Palabras clave: ventilación mecánica, complicaciones.)

\section{Referencias}

1. Hibermann $M$ : The evolution of intensive care units. Crit Care Med 1975: 3: 1.59-165.

2. Cerda $M$. Soavedra $R$. Aspillaga $M$. et al.: Ventilación mectinica en insuficiencia respiratoria de origen pulmonar. Rev Chil Pediatr 1984; $55: 25-28$.

3. Klamburg J, Latorre F, Porta $f$, et al.: Complicaciones de la ventilación mecánica. Estudjo prospectivo de 698 episodios consecutivos. Med Intensiva 1981; 5 : $175 \cdot 184$.

4. Klamburg $J$ : Morbilidad y mortalidad en la ventilación mecánica prolongada. Med Intensiva 1983; 7 : 205-225.

5. Rivera $R$. Tibballs $J$ : Complications of endotracheal inlubation and mechanical ventilation ininfants and children. Crit Care Med 1992; 20: 193-199.

6. Zwillich C. Pierson D, Greagh $C$. et al.: Complications of assisted ventilation. A prospective study of $354 \mathrm{con}$. secutive episodes. Am J Med 1974; 57: 161-169.

7. Strieter $R$, Lynch $f$ : Complications in the ventilated patients. Clinics in chest Medicine 1988; 9: 127-139.

8. Fnunn J, Milledge J. Singaraya J: Survival of patients ventilaced in an incensive therapy unit. Br Med J 1979; 9: $1525-1527$. 